\title{
Pacific
}

Journal of

Mathematics

\section{A REFLECTION PRINCIPLE IN COMPLEX SPACE FOR A CLASS OF HYPERSURFACES AND MAPPINGS}

Francine ANTOINETte MEYLAN 


\title{
A REFLECTION PRINCIPLE IN COMPLEX SPACE FOR A CLASS OF HYPERSURFACES AND MAPPINGS
}

\section{FranCine MEYLAN}

\begin{abstract}
The Schwarz reflection principle in one complex variable can be stated as follows. Let $M$ and $M^{\prime}$ be two real analytic curves in $\mathbb{C}$ and $\mathcal{H}$ a holomorphic function defined on one side of $M$, extending continuously through $M$, and mapping $M$ into $M^{\prime}$. Then $\mathcal{H}$ has a holomorphic extension across $M$. We address here the question of extending this classical theorem to higher complex dimensions for some class of hypersurfaces and mappings.
\end{abstract}

1. Introduction and main results. Let $M$ and $M^{\prime}$ be two germs of real analytic hypersurfaces at 0 in $\mathbb{C}^{n+1}, n \geq 1$, and $\mathcal{H}$ a holomorphic mapping defined on one side of $M$, extending smoothly up to $M$, and mapping $M$ into $M^{\prime}$, with $\mathcal{H}(0)=0$. We say that the reflection principle holds if $\mathcal{H}$ extends holomorphically across $M$ at 0 . In the complex plane, by the classical Schwarz reflection principle, the reflection principle holds. The first results in higher dimension were due to $H$. Lewy [16] and S. Pincuk [18]. They proved independently that the reflection principle holds if $M$ and $M^{\prime}$ are strictly pseudoconvex, and $\mathcal{H}$ is a diffeomorphism from $M$ to $M^{\prime}$. Other results on the reflection principle have been obtained by Baouendi, Jacobowitz and Treves [2], Baouendi and Rothschild [3], [4], [5], Bell [6], Diederich and Fornaess [10], Diederich and Webster [11], as well as by other mathematicians. In [3] and [4] the authors obtain a reflection principle for $M$ and $M^{\prime}$ germs of real analytic hypersurfaces at 0 , of finite type, satisfying an algebraic condition. The mapping they consider is of finite multiplicity. In [5] the authors consider the case of $\mathbb{C}^{2}$ and obtain a more general result which allows $M$ and $M^{\prime}$ to be of infinite type; in fact they obtain a necessary and sufficient condition for the reflection principle to hold. In this paper, we address the question of extending the reflection principle in $\mathbb{C}^{n+1}, n \geq 1$, to a new class of germs of real analytic hypersurfaces allowing them to be of infinite type, and to a new 
class of mappings, generalizing the results obtained in [3], [4], and [5].

To make this more precise, we first introduce notation and definitions needed in the sequel. Let $M$ be a germ of a real analytic hypersurface at 0 . After a local holomorphic change of coordinates, we can assume that there exists $\Omega$, a sufficiently small open neighborhood of 0 in $\mathbb{C}^{n+1}, n \geq 1$, such that $M$ is given in $\Omega$ by

$$
\operatorname{Im} w=\varphi(z, \bar{z}, \operatorname{Re} w),
$$

with $z \in \mathbb{C}^{n}, w \in \mathbb{C}, \varphi$ a real valued convergent power series and $\varphi(z, 0, w) \equiv 0$. Such a choice of coordinates is called normal coordinates.

Let $\Omega^{+}=\{(z, w) \in \Omega \mid \operatorname{Im} w>\varphi(z, \bar{z}, \operatorname{Re} w)\}$, and similarly $\overline{\Omega^{+}}=\{(z, w) \in \Omega \mid \operatorname{Im} w \geq \varphi(z, \bar{z}, \operatorname{Re} w)\}$. Consider a mapping $\mathcal{H}$ holomorphic in $\Omega^{+}$, smooth in $\overline{\Omega^{+}}$, valued in $\mathbb{C}^{n+1}$ and satisfying $\mathcal{H}(M) \subset M^{\prime}$, where $M^{\prime}$ is another germ of a real analytic hypersurface at 0 in $\mathbb{C}^{n+1}$, also given in normal coordinates $\left(z^{\prime}, w^{\prime}\right)$. We shall always assume $\mathcal{H}(0)=0$. We shall say that $\operatorname{such}\left(M, M^{\prime}, \mathcal{H}\right)$ satisfy the hypothesis of the reflection principle.

Write $\mathcal{H}=\left(\mathcal{F}_{1}, \mathcal{F}_{2}, \ldots, \mathcal{F}_{n}, \mathcal{G}\right)=(\mathcal{F}, \mathcal{G})$ and denote by $\left(F_{1}, F_{2}\right.$, $\left.\ldots, F_{n}, G\right)=(F, G)$ the formal holomorphic Taylor series of the components $\mathcal{F}_{1}, \mathcal{F}_{2}, \ldots, \mathcal{F}_{n}, \mathcal{G}$ at 0 . Let $H=\left(f_{1}, \ldots, f_{n}, g\right)$ be the restriction of $\mathcal{H}$ to $M$. Recall that $M$ is flat if after a holomorphic change of coordinates in $\mathbb{C}^{n+1}, M$ is given by $\operatorname{Im} w=0$.

Let $M$ be a germ of a real analytic hypersurface given in normal coordinates by

$$
\operatorname{Im} w=\varphi(z, \bar{z}, \operatorname{Re} w)=(\operatorname{Re} w)^{m} \tilde{\varphi}(z, \bar{z}, \operatorname{Re} w),
$$

where $\tilde{\varphi}$ is a real valued convergent power series in $z, \bar{z}, \operatorname{Re} w$ such that $\tilde{\varphi}(z, \bar{z}, 0) \not \equiv 0$ and $m \geq 0$. We shall see that $m$ is independent of the choice of normal coordinates. Write

$$
\bar{\varphi}(z, \zeta, 0)=\sum_{\alpha} a_{\alpha}(z) \zeta^{\alpha} .
$$

Note that $m=0$ if and only if $M$ is of finite type in the sense of $[\mathbf{8}],[\mathbf{1 5}]$. We introduce the following definition.

DEFINITION 1.4. $M$ is $m$-essential at 0 if the ideal $\left(a_{\alpha}(z)\right)$ in the ring of formal power series $\mathbb{C}[[z]]$ generated by all the $a_{\alpha}(z)$ is 
of finite codimension, i.e.

$$
m \text { - ess.type } M=\operatorname{dim}_{\mathbb{C}} \mathbb{C}[[z]] /\left(a_{\alpha}(z)\right)<\infty .
$$

Note that $M$ is 0 -essential at 0 if and only if $M$ is essentially finite in the sense of [2], [3]. Also we shall see that the above definition is independent of the choice of normal coordinates. Recall the following definitions:

Let $\left(M, M^{\prime}, \mathcal{H}\right)$ satisfy the hypothesis of the reflection principle. $\mathcal{H}$ is not totally degenerate at 0 if

$$
\operatorname{det}\left(\frac{\partial F_{j}}{\partial z_{k}}(z, 0)\right) \not \equiv 0, j, k=1, \ldots, n .
$$

Also, $\mathcal{H}$ is of finite multiplicity at 0 if

$$
\text { mult. } \mathcal{H}=\operatorname{dim}_{\mathbb{C}} \mathbb{C}[[z]] /(F(z, 0))<\infty .
$$

Note that 1.7 implies 1.6 by standard algebra ([12]). It is known that these two definitions are independent of the choice of normal coordinates $([\mathbf{3}])$. For germs of real analytic hypersurfaces which are $m$-essential at 0 , we have the following theorems which extend the results obtained in [3] and [4].

THEOREM 1. Let $\left(M, M^{\prime}, \mathcal{H}\right)$ satisfy the hypothesis of the reflection principle. Then $\mathcal{H}$ extends holomorphically to a neighborhood of 0 in $\mathbb{C}^{n+1}$, if one of the following conditions holds:

(1) $M$ is $m$-essential at $0, G \not \equiv 0$, and $\mathcal{H}$ not totally degenerate at 0 .

(2) $M^{\prime}$ is $m^{\prime}$-essential at $0, G \not \equiv 0$, and $\mathcal{H}$ of finite multiplicity at 0.

(3) $M^{\prime}$ is $m^{\prime}$-essential at $0, G \not \equiv 0$ and $\mathcal{H}$ not totally degenerate at 0 .

Write

$$
G(z)=\sum_{j=k_{0}}^{\infty} G_{j}(z) w^{j}
$$

with $k_{0}$ minimal so that $G_{k_{0}}(z) \not \equiv 0$. We shall see that $k_{0}$ is independent of the choice of normal coordinates. 
THEOREM 2. Let $\left(M, M^{\prime}, \mathcal{H}\right)$ satisfy the hypothesis of the reflection principle. If either

(1) $M$ is $m$-essential at $0, G \not \equiv 0, \mathcal{H}$ is not totally degenerate at 0 , or

(2) $M^{\prime}$ is $m^{\prime}$-essential at $0, G \not \equiv 0$, and $\mathcal{H}$ is of finite multiplicity at 0 ,

then

$$
m \text { - ess. type } M=(\text { mult. } \mathcal{H})\left(m^{\prime}-\text { ess. type } M^{\prime}\right)
$$

with all three integers finite, and

$$
m-1=k_{0}\left(m^{\prime}-1\right) .
$$

Let $\left(M, M^{\prime}, \mathcal{H}\right)$ satisfy the hypothesis of the reflection principle. Write

$$
F(z, w)=\sum_{j=0}^{\infty} F_{j}^{*}(z) w^{j},
$$

where $F_{j}^{*}(z)=\left(F_{j 1}^{*}(z), F_{j 2}^{*}(z), \ldots, F_{j n}^{*}(z)\right)$ are formal power series in $z$.

Define $l$ to be minimal such that

$$
F_{l}^{*}(z) \not \equiv F_{l}^{*}(0)
$$

We shall see that $l$ is independent of the choice of normal coordinates if $M$ is not flat, $M^{\prime}$ is of infinite type and $G \not \equiv 0$. We introduce the following definition.

Definition 1.13. Let $\left(M, M^{\prime}, \mathcal{H}\right)$ satisfy the hypothesis of the reflection principle, with $M$ not flat, $M^{\prime}$ of infinite type, and $G \not \equiv 0$. We say that $\mathcal{H}$ is l-tangentially finite at 0 if

$$
\text { (1.14) } l \text { - tang.mult. } \mathcal{H}=\operatorname{dim}_{\mathbb{C}} \mathbb{C}[[z]] /\left(F_{l}^{*}(z)-F_{l}^{*}(0)\right)<\infty \text {. }
$$

Note that $\mathcal{H}$ is 0 -tangentially finite if and only if $\mathcal{H}$ is of finite multiplicity. We shall see that the above definition is independent of the choice of normal coordinates. For $\mathcal{H} l$-tangentially finite, we get the following extension result. 
THEOREM 3. Let $\left(M, M^{\prime}, \mathcal{H}\right)$ satisfy the hypothesis of the reflection principle, with $M^{\prime}$ of infinite type. Then $\mathcal{H}$ extends holomorphically to a neighborhood of 0 in $\mathbb{C}^{n+1}, n \geq 1$, if any one of the following conditions holds

(1) $M$ is non flat, $M^{\prime}$ is $m^{\prime}$-essential, $\mathcal{H}$ is l-tangentially finite and $G \not \equiv 0$.

(2) $M$ is non flat, $M^{\prime}$ is $m^{\prime}$-essential, $\operatorname{det}\left(\frac{\partial F_{l j}^{*}}{\partial z_{k}}(z)\right) \not \equiv 0$ and $G \not \equiv 0$.

As for mappings of finite multiplicity, there exists a relationship between $m$-ess.type $M$ and $l$-tang.mult. $\mathcal{H}$. Indeed we have:

THEOREM 4. Let $\left(M, M^{\prime}, \mathcal{H}\right)$ satisfy the hypothesis of the reflection principle, with $M$ m-essential at $0, M^{\prime}$ of infinite type, $G \not \equiv 0, \operatorname{det}\left(\frac{\partial F_{l j}^{*}}{\partial z_{k}}(z)\right) \not \equiv 0, j, k=1, \ldots, n$. Then $\mathcal{H}$ is l-tangentially finite and

$(l$ - tang. mult. $\mathcal{H})$ divides $(m-$ ess. type $M)$.

Note that under the assumptions of Theorem $4, M^{\prime}$ need not be $m^{\prime}$-essential at 0 as it is shown in the following example.

Example 1.15. Consider, in $\mathbb{C}^{3}, M$ given by

$$
w-\bar{w}=2 i|w|^{6}\left(\left|z_{1}\right|^{2}+\left|z_{2}\right|^{2}\right)+2 i|w|^{18}\left|z_{1}\right|^{16}
$$

$M^{\prime}$ given by

$$
\begin{gathered}
w^{\prime}-\bar{w}^{\prime}=2 i\left|w^{\prime}\right|^{2}\left|z_{1}^{\prime}\right|^{16}+2 i\left|w^{\prime}\right|^{4}\left(\left|z_{1}^{\prime}\right|^{2}+\left|z_{2}^{\prime}\right|^{2}\right) \\
\mathcal{F}_{1}(z, w)=z_{1} w, \mathcal{F}_{2}(z, w)=z_{2} w, \mathcal{G}(z, w)=w .
\end{gathered}
$$

Here $M$ is 6-essential but $M^{\prime}$ is not $m^{\prime}$-essential.

REMARK 1.16. Our proof of part (3) of Theorem 1 in the finite type case is different from that given in [3] and [4]. The proof of Theorem 3 for $\mathbb{C}^{2}$ is also different from that given in [5].

Section 2 deals with invariants associated to germs of real analytic hypersurfaces and holomorphic maps; we introduce new numerical invariants associated to germs of real analytic hypersurfaces and 
holomorphic maps. In Section 3, we give the proofs of Theorem 1 and Theorem 3. The proofs of Theorem 2 and Theorem 4 are given in Section 4.

The results of this paper were part of the author's Ph.D. dissertation at the University of California, San Diego. The author wishes to thank Salah Baouendi and Linda Rothschild for their help and support during the completion of this work.

2. Invariants associated to germs of real analytic hypersurfaces and holomorphic maps. Let $M$ be a germ of a real analytic hypersurface given in normal coordinates by 1.1. Put $w=$ $s+i t$. We have the following lemma:

LEMMA 2.1. The integer $k_{0}$ defined by (1.8) is independent of the choice of normal coordinates.

This is easily shown by observing from the definition of normal coordinates that we have $G(z, w)=w G^{1}(z, w)$, with $G^{1}(z, w)$ another formal power series.

Proposition 2.2. Let $\left(M, M^{\prime}, \mathcal{H}\right)$ satisfy the hypothesis of the reflection principle, with $M^{\prime}$ of infinite type, and $G \not \equiv 0$. Then the following is true:

(1) $G_{k_{0}}(z) \equiv G_{k_{0}}(0) \in \mathbb{R}-\{0\}$.

(2) If $\mathcal{H}$ is not totally degenerate, then

$$
m-1=k_{0}\left(m^{\prime}-1\right)
$$

where $m$ and $m^{\prime}$ are defined by (1.2).

We have the following corollary:

Corollary 2.3. The integer $m$ defined by (1.2) is independent of the choice of normal coordinates.

The proof is immediate by using (2), since $k_{0}=1$ in this case.

Proof of Proposition 2.2. Applying Proposition 3.16 of [4], we obtain that $M$ is of infinite type. Let $M$ given by 1.2 and $M^{\prime}$ given by $t^{\prime}=s^{\prime m^{\prime}} \psi\left(z^{\prime}, \bar{z}^{\prime}, s^{\prime}\right)$. Write $G(z, w)=w^{k_{0}} G^{k_{0}}(z, w)$, with $k_{0} \geq 1$. 
As $\mathcal{H}(M) \subset M^{\prime}$, we get

$$
\begin{aligned}
(s+ & \left.i s^{m} \tilde{\varphi}(z, \bar{z}, s)\right)^{k_{0}} G^{k_{0}}-\left(s-i s^{m} \tilde{\varphi}(z, \bar{z}, s)\right)^{k_{0}} \overline{G^{k_{0}}} \\
\equiv & 2 i\left(\frac{\left(s+i s^{m} \tilde{\varphi}(z, \bar{z}, s)\right)^{k_{0}} G^{k_{0}}+\left(s-i s^{m} \tilde{\varphi}(z, \bar{z}, s)\right)^{k_{0}} \overline{G^{k_{0}}}}{2}\right)^{m^{\prime}} \\
& \cdot \psi\left(F, \bar{F}, \frac{G+\bar{G}}{2}\right),
\end{aligned}
$$

where

$$
G^{k_{0}}=G^{k_{0}}\left(z, s+i s^{m} \tilde{\varphi}(z, \bar{z}, s)\right) \text { and } \bar{G}^{k_{0}}=\bar{G}^{k_{0}}\left(\bar{z}, s-i s^{m} \tilde{\varphi}(z, \bar{z}, s)\right) \text {. }
$$

Using the binomial formula, we can rewrite 2.4 as

$$
\begin{aligned}
& s^{k_{0}}\left(G^{k_{0}}-\bar{G}^{k_{0}}\right)+c s^{m+k_{0}-1} \tilde{\varphi}\left[G^{k_{0}}+\bar{G}^{k_{0}}+\alpha(z, \bar{z}, s)\right] \\
& \quad=c^{\prime} s^{k_{0} m^{\prime}}\left[G^{k_{0}}+\bar{G}^{k_{0}}+\beta(z, \bar{z}, s)\right]^{m^{\prime}} \psi\left(F, \bar{F}, \frac{G+\bar{G}}{2}\right),
\end{aligned}
$$

with $c, c^{\prime}$ constants $\neq 0$.

$$
\alpha(0, \bar{z}, s) \equiv \alpha(z, 0, s) \equiv 0, \beta(0, \bar{z}, s) \equiv \beta(z, 0, s) \equiv 0 .
$$

Dividing 2.5 by $s^{k_{0}}$, and putting $s=0, \bar{z}=0$, we obtain (1). In order to prove (2), we first assume that $m+k_{0}-1<k_{0} m^{\prime}$. Differentiating $2.5 m+k_{0}-1$ times with respect to $s$ and putting $s=0$, we get

$$
\begin{aligned}
\frac{\partial^{m-1} G^{k_{0}}}{\partial w^{m-1}}(z, 0)- & \frac{\overline{\partial^{m-1} G^{k_{0}}}}{\partial w^{m-1}}(\bar{z}, 0) \\
& +C \tilde{\varphi}\left(G^{k_{0}}(z, 0)+\overline{G^{k_{0}}}(\bar{z}, 0)+\alpha(z, \bar{z}, 0)\right) \equiv 0,
\end{aligned}
$$

with $C$ constant $\neq 0$.

Using (2.6) and the fact that we work in normal coordinates, we get that $\tilde{\varphi}(z, \bar{z}, 0) \equiv 0$, which is impossible by 1.2 .

Suppose that $m+k_{0}-1>k_{0} m^{\prime}$. Differentiating $2.5 k_{0} m^{\prime}$ times with respect to $s$ and putting $s=0$, we get

$$
\begin{aligned}
& \frac{\partial^{k_{0} m^{\prime}-k_{0}} G^{k_{0}}}{\partial w^{k_{0} m^{\prime}-k_{0}}}(z, 0)-\frac{\overline{\partial^{k_{0} m^{\prime}-k_{0}} G^{k_{0}}}}{\partial w^{k_{0} m^{\prime}-k_{0}}}(\bar{z}, 0) \\
& \equiv C^{\prime}\left(G^{k_{0}}(z, 0)+\overline{G^{k_{0}}}(\bar{z}, 0)\right. \\
& \quad+\beta(z, \bar{z}, 0))^{m^{\prime}} \psi(F(z, 0), \bar{F}(\bar{z}, 0), 0),
\end{aligned}
$$


with $C^{\prime}$ a constant $\neq 0$.

Using 2.6 and the fact that we work in normal coordinates, we get that

$$
\psi(F(z, 0), \bar{F}(\bar{z}, 0), 0) \equiv 0 .
$$

Since $\mathcal{H}$ is not totally degenerate by assumption, it is easily shown, differentiating 2.7 with respect to $z_{k}, k=1, \ldots, n$, and using Cramer's rule, that 2.7 is impossible. Hence we get the desired equation (2) of Proposition 2.2.

We have the following proposition:

Proposition 2.8. Let $\left(M, M^{\prime}, \mathcal{H}\right)$ satisfy the hypothesis of the reflection principle, with $M^{\prime}$ of infinite type, $G \not \equiv 0$ and $\mathcal{H}$ not totally degenerate. Then $G_{k_{0}}, G_{k_{0}+1}, \ldots, G_{k_{0}+m-1}$ are constant and real.

Proof. Dividing 2.5 by $s^{k_{0}}$, we get

$$
\begin{aligned}
& \left(G^{k_{0}}-\bar{G}^{k_{0}}\right)+c s^{m-1} \tilde{\varphi}\left[G^{k_{0}}+\bar{G}^{k_{0}}+\alpha(z, \bar{z}, s)\right] \\
& \quad=c^{\prime} s^{m-1}\left[G^{k_{0}}+\bar{G}^{k_{0}}+\beta(z, \bar{z}, s)\right]^{m^{\prime}} \psi\left(F, \bar{F}, \frac{G+\bar{G}}{2}\right) .
\end{aligned}
$$

Differentiating $2.9 j$ times with respect to $s, j \leq m-1$, and putting $s=0$, we get

$$
\begin{array}{r}
G^{k_{0}}(z, 0)-\bar{G}^{k_{0}}(\bar{z}, 0) \equiv 0 \\
\vdots \\
\frac{\partial^{m-1} G^{k_{0}}}{\partial w^{m-1}}(z, 0)-\frac{\overline{\partial^{m-1} G^{k_{0}}}}{\partial w^{m-1}}(\bar{z}, 0) \equiv 0
\end{array}
$$

as we work in normal coordinates. Putting $\bar{z}=0$ in these equations, we get the desired conclusion.

Corollary 2.10. If $\mathcal{H}$ is a local biholomorphism at 0 , and $M^{\prime}$ is of infinite type, then $G_{1}, \ldots, G_{m}$ are constant and real.

We have the following proposition:

Proposition 2.11. Let $\left(M, M^{\prime}, \mathcal{H}\right)$ satisfy the hypothesis of the reflection principle, with $M m$-essential, $G \not \equiv 0$ and $\mathcal{H}$ not totally degenerate. Then $M^{\prime}$ is $m^{\prime}$-essential, $\mathcal{H}$ is of finite multiplicity and

$$
m \text { - ess. type } M=(\text { mult. } \mathcal{H})\left(m^{\prime}-\text { ess. type } M^{\prime}\right) .
$$


Before giving the proof, we state the following corollary:

Corollary 2.13. Definition 1.4 and the number defined by 1.5 is independent of the choice of normal coordinates.

The proof of the Corollary is immediate from Proposition 2.11. Note that, unlike the finite type case, the conditions $M m$-essential and $G \not \equiv 0$ are not enough to guarantee $\mathcal{H}$ not totally degenerate, as it is shown in the following example (considered in [5] for another purpose).

EXAMPLE 2.14. Consider in $\mathbb{C}^{2} \mathcal{F}(z, w)=(1+z) w, \mathcal{G}(z, w)=$ $-z(1+z) w^{3}, M^{\prime}$ given by $w^{\prime}-\bar{w}^{\prime}=z^{\prime} \bar{z}^{\prime 2}-\bar{z}^{\prime} z^{\prime 2}$, and $M$ given by $t=s \psi(z, \bar{z})$, with $\psi(z, 0) \equiv \psi(0, \bar{z}) \equiv 0$, and $\psi$ chosen such that $\mathcal{H}=(\mathcal{F}, \mathcal{G})$ maps $M$ into $M^{\prime}$. Here, we have $M$ is 1-essential, $G \not \equiv 0, M^{\prime}$ is 0 -essential, but $\mathcal{H}$ is totally degenerate.

Proof of Proposition 2.11. The case $m=0$ has been considered in [3] and [4]. Assume $m>0$. By Proposition 3.28 in [4], we have $m^{\prime}>0$. Differentiating $2.5 m+k_{0}-1=k_{0} m^{\prime}$ times with respect to $s$, and putting $s=0$, we obtain

$$
\begin{aligned}
& C \tilde{\varphi}(z, \bar{z}, 0)\left(G^{k_{0}}(z, 0)+\overline{G^{k_{0}}}(\bar{z}, 0)+\alpha(z, \bar{z}, 0)\right) \\
& \equiv C^{\prime}\left(G^{k_{0}}(z, 0)+\overline{G^{k_{0}}}(\bar{z}, 0)\right. \\
& \quad+\beta(z, \bar{z}, 0))^{m^{\prime}} \psi(F(z, 0), \bar{F}(\bar{z}, 0), 0), C, C^{\prime} \text { constants } \neq 0 .
\end{aligned}
$$

Using 2.6 and (1) of proposition 2.2 , we can rewrite 2.15 as

$$
h(z, \bar{z}) \varphi(z, \bar{z}, 0) \equiv \psi(F(z, 0), \bar{F}(\bar{z}, 0), 0),
$$

where $h(z, \bar{z})$ is a formal power series with $h(0) \neq 0$. Inspecting the proof of Theorem 3 in [3], which uses tools of commutative algebra, we conclude that $\mathcal{H}$ is of finite multiplicity, $M^{\prime}$ is $m^{\prime}$-essential and that 2.12 holds. This completes the proof of Proposition 2.11.

We denote by $L_{k}, k=1, \ldots, n$, the antiholomorphic vector fields tangent to $M$ given by

$$
L_{k}=\frac{\partial}{\partial \bar{z}_{k}}-i \frac{\varphi_{\bar{z}_{k}}}{1+i \varphi_{s}} \frac{\partial}{\partial s} .
$$

Let $l$ be defined by 1.12 . We have the following propositions: 
Proposition 2.17. Let $\left(M, M^{\prime}, \mathcal{H}\right)$ satisfy the hypothesis of the reflection principle, with $G \not \equiv 0$ and $M^{\prime}$ of infinite type at 0 . Then we have:

$$
m>2 l
$$

$$
G_{k_{0}+j}(z) \equiv G_{k_{0}+j}(0), 0 \leq j \leq l
$$

Proof. The proof is similar to that of 2.5 and 2.6 in [5], and is left to the reader.

Proposition 2.20. Let $\left(M, M^{\prime}, \mathcal{H}\right)$ satisfy the hypothesis of the reflection principle, with $G \not \equiv 0, M$ not flat and $M^{\prime}$ of infinite type at 0 . Then

$$
\begin{aligned}
& f(z, \bar{z}, s)=s^{p} \tilde{f}_{0}(z, \bar{z}, s), \\
& \bar{L}_{j} f(z, \bar{z}, s)=s^{l} \tilde{f}_{j}(z, \bar{z}, s), j=1, \ldots, n,
\end{aligned}
$$

where $p$ is minimal such that $F_{p}^{*}(z) \not \equiv 0, \tilde{f}_{0}=\left(\tilde{f}_{01}, \tilde{f}_{02}, \ldots, \tilde{f}_{0 n}\right)$, with $\tilde{f}_{0}$ smooth, and $\tilde{f}_{j}=\left(\tilde{f}_{j 1}, \tilde{f}_{j 2}, \ldots, \tilde{f}_{j n}\right)$, with $\tilde{f}_{j}$ smooth.

Furthermore, there exists an index $j_{0}$ such that

$$
\bar{L}_{j 0} f(z, \bar{z}, s)=s^{l} \tilde{f}_{j 0}(z, \bar{z}, s),
$$

with $\tilde{f}_{j 0}(z, \bar{z}, 0) \not \equiv 0$.

Proof. The proof is similar to that of Theorem 3 in [5] and is left to the reader.

Proposition 2.23. Let $\left(M, M^{\prime}, \mathcal{H}\right)$ satisfy the hypothesis of the reflection principle, with $M$ not flat, $M^{\prime}$ of infinite type at 0 and $G \not \equiv 0$, Then the number $l$ is independent of the choice of normal coordinates.

Proof. Let $H=\left(f_{1}, f_{2}, \ldots, f_{n}, g\right)$ and consider $H^{*}$, the pushforward of tangent vectors from $M$ to $M^{\prime}$. We have for $i=1, \ldots, n$,

$$
H^{*}\left(L_{i z, s}\right)=\sum_{j=1}^{n} c_{i j}(z, \bar{z}, s) L_{j H(z, \bar{z}, s)}^{\prime}
$$


with $c_{i j}$ smooth. Write $\left(c_{i j}(z, \bar{z}, s)\right)=s_{\alpha}\left(\tilde{c}_{i j}(z, \bar{z}, s)\right)$ with $\tilde{c}_{i j}$ smooth, $\left(\tilde{c}_{i j}(z, \bar{z}, 0)\right) \not \equiv(0)$. Using standard tools of linear algebra, it is easily shown that $\alpha$ is independent of the choice of normal coordinates. Using the chain rule, it is easy to show that $\left(c_{i j}(z, \bar{z}, s)\right)=$ $\left(L_{i} \bar{f}_{j}(z, \bar{z}, s)\right)$. Hence, by 2.22 , we conclude that the number $l$ is independent of the choice of normal coordinates.

REMARK 2.24. It is easily shown that the number $l$ is also an invariant if $M$ is of finite type and $G \not \equiv 0$. It would be interesting to know whether $l$ is again a biholomorphic invariant in the case $M$ of infinite type, $M^{\prime}$ of finite type and $G \not \equiv 0$. It should be noted as shown in Theorem 2 in [5] that $l$ is an invariant in $\mathbb{C}^{2}$ for this case. Also, if $k_{0}=\infty$, i.e. $G \equiv 0$, then $l$ is not a biholomorphic invariant, even for the $\mathbb{C}^{2}$ case, as it is shown in Remark 2.30 in [5].

We have the following proposition:

Proposition 2.25. Let $\left(M, M^{\prime}, \mathcal{H}\right)$ satisfy the hypothesis of the reflection principle, where $M$ is not flat, $M^{\prime}$ of infinite type at 0 and $G \not \equiv 0$. Then the number defined by (1.14) is independent of the choice of normal coordinates.

Proof. We have $F_{l}^{*}(z)=l ! \frac{\partial^{l} F}{\partial w^{l}}(z, 0)$. The case $l=0$ has been considered in [3]. Let $l \geq 1$. Considrer $\theta:(z, w) \rightarrow(\tilde{z}, \tilde{w})$ a holomorphic change of normal coordinates in the source, and let $\tilde{F}=F \circ \theta^{-1}$. By Proposition 2.23, we have to compare

$$
\frac{\partial^{l} F}{\partial w^{l}}(z, 0) \text { and } \frac{\partial^{l} \tilde{F}}{\partial \tilde{w}^{l}}(\tilde{z}, 0) \text {. }
$$

We have

$$
\begin{aligned}
\frac{\partial^{l} F}{\partial w^{l}}(z, 0)= & \frac{\partial^{l-1}}{\partial w^{l-1}}\left(\frac{\partial \tilde{F}(\tilde{z}, \tilde{w})}{\partial w}\right)(z, 0) \\
= & \frac{\partial^{l-1}}{\partial w^{l-1}}\left(\frac{\partial \tilde{F}}{\partial \tilde{w}}(\tilde{z}, \tilde{w}) \frac{\partial \tilde{w}}{\partial w}(z, w)\right. \\
& \left.+\sum_{j=1}^{n} \frac{\partial \tilde{F}}{\partial \tilde{z}}(\tilde{z}, \tilde{w}) \frac{\partial \tilde{z}_{j}}{\partial w}(z, w)\right)(z, 0) .
\end{aligned}
$$


By definition of $l$, we have that 2.26 is of the form $\frac{\partial^{l} \tilde{F}}{\partial \tilde{w}^{l}}(\tilde{z}, 0)$ . $\left(\frac{\partial \tilde{w}}{\partial w}(z, 0)\right)^{l}+$ a sum of terms which are product of $\frac{\partial^{k} \tilde{F}}{\partial \tilde{w}^{k}}(\tilde{z}, 0)$ and $\frac{\partial^{\alpha} \tilde{w}}{\partial w^{\alpha}}(z, 0), 1 \leq \alpha \leq l, 1 \leq k \leq l-1$. Using Corollary 2.10 and 2.18 , we conclude that

$$
\frac{\partial^{l} F}{\partial w^{l}}(z, 0)-\frac{\partial^{l} F}{\partial w^{l}}(0,0)=C\left(\frac{\partial^{l} \tilde{F}}{\partial \tilde{w}^{l}}(\tilde{z}, 0)-\frac{\partial^{l} \tilde{F}}{\partial \tilde{w}^{l}}(0,0)\right),
$$

with $C$ constant $\neq 0$. This completes the proof for a holomorphic change of normal coordinates in the source.

Consider $\kappa^{-1}:\left(z^{\prime}, w^{\prime}\right) \rightarrow\left(\tilde{z}^{\prime}, \tilde{w}^{\prime}\right)$ a holomorphic change of normal coordinates in the target space, and let $\tilde{H}=\kappa^{-1} \circ H$. We have $F_{j}(z, w)=\kappa_{j} \circ \tilde{H}(z, w)$, where $\kappa=\left(\kappa_{1}, \kappa_{2}, \ldots, \kappa_{n+1}\right)$. As $l \geq 1$, we have

$$
\tilde{H}(z, 0)=\tilde{H}(0,0)=0 .
$$

We get

$$
\begin{aligned}
\frac{\partial^{l} F_{j}}{\partial w^{l}}(z, 0)= & \frac{\partial^{l}\left(\kappa_{j} \circ \tilde{H}\right)}{\partial w^{l}}(z, 0) \\
= & \frac{\partial^{l-1}}{\partial w^{l-1}}\left(\sum_{k=1}^{n} \frac{\partial \kappa_{j}}{\partial \tilde{z}_{k}^{\prime}}(\tilde{H}(z, w)) \frac{\partial \tilde{F}_{k}}{\partial w}\right. \\
& \left.+\frac{\partial \kappa_{j}}{\partial \tilde{w}^{\prime}}(\tilde{H}(z, w)) \frac{\partial \tilde{G}}{\partial w}\right)(z, 0) .
\end{aligned}
$$

Using the definition of $l, 2.19$ and 2.27 , we obtain that 2.28 is of the form

$$
C^{\prime}+\sum_{k=1}^{n} \frac{\partial \kappa_{j}}{\partial \tilde{z}_{k}^{\prime}}(0) \frac{\partial^{l} \tilde{F}_{k}}{\partial w^{l}}(z, 0)
$$

with $C^{\prime}$ constant. Therefore

$$
\frac{\partial^{l} F_{j}}{\partial w^{l}}(z, 0)-\frac{\partial^{l} F_{j}}{\partial w^{l}}(0,0)=\left(\frac{\partial \kappa_{j}}{\partial \tilde{z}_{k}^{\prime}}(0)\right)\left(\frac{\partial^{l} \tilde{F}_{k}}{\partial w^{l}}(z, 0)-\frac{\partial^{l} \tilde{F}_{k}}{\partial w^{l}}(0,0)\right) .
$$

As we work in normal coordinates, $\operatorname{det}\left(\frac{\partial \kappa_{j}}{\partial \tilde{z}_{k}^{\prime}}(0)\right) \neq 0$. This completes the proof in the case of a holomorphic change of normal coordinates in the target. 
3. Proof of Theorem 1 and Theorem 3. Let $\alpha=\left(\alpha_{1}, \ldots, \alpha_{n}\right)$ $\in \mathbb{Z}^{n}$. Define $L^{\alpha}$ to be $L^{\alpha}=L_{1}{ }^{\alpha_{1}} L_{2}{ }^{\alpha_{2}} \ldots L_{n}{ }^{\alpha_{n}}$. We have the following propositions:

Proposition 3.1. Let $\left(M, M^{\prime}, \mathcal{H}\right)$ satisfy the hypothesis of the reflection principle. Then the following is true:

(1) If $\mathcal{H}$ is not totally degenerate at 0 , then there exists a multiindex $\alpha_{0}$ such that

$$
L^{\alpha_{0}}\left(\operatorname{det} L_{j} \bar{f}_{k}\right)(0) \neq 0, j, k=1, \ldots, n .
$$

(2) If $M$ is not flat, $M^{\prime}$ is of infinite type at $0, G \not \equiv 0$ and $\operatorname{det}\left(\frac{\partial F_{l j}^{*}}{\partial z_{k}}(z)\right) \not \equiv 0$, then there exists a multi-index $\beta_{0}$ such that

$$
L^{\beta_{0}}\left(\operatorname{det} \overline{\tilde{f}_{j k}}\right)(0) \neq 0, j, k=1, \ldots, n,
$$

where $\overline{\tilde{f}_{j k}}$ is given by (2.21). Furthermore, if

$$
D(z, \bar{z}, s)=\operatorname{det} L_{j} \bar{f}_{k}(z, \bar{z}, s),
$$

then for every multi-index $\alpha$,

$$
L^{\alpha} D(z, \bar{z}, s)=s^{n l} D_{\alpha}(z, \bar{z}, s),
$$

with $D_{\alpha}(\dot{z}, \bar{z}, s)$ smooth and $D_{\beta_{0}}(0) \neq 0$.

Proof of (1). See Proposition 3.18 of [3].

Proof of (2). We can assume $l \geq 1$. Let $M$ be given in normal coordinates by 1.2 . By 2.21 , we have

$$
\bar{f}_{k}(z, \bar{z}, s)=s^{p} \overline{\tilde{f}_{0 k}}(z, \bar{z}, s),
$$

with $\overline{\tilde{f}_{0 k}}$ smooth, $k=1, \ldots, n$. We claim that $s^{p-l} L_{j} \overline{\tilde{f}_{0 k}}$ is smoothand

$$
\overline{\tilde{f}_{j k}}(z, \bar{z}, 0)=\left(\frac{L_{j} \overline{\tilde{f}_{0 k}}}{s^{l-p}}\right)(z, \bar{z}, 0)
$$


Indeed, using $2.18,2.21$, and 3.5 , we obtain

$$
\begin{aligned}
L_{j} \bar{f}_{k}(z, \bar{z}, s) & =s^{l} \overline{\tilde{f}_{j k}}(z, \bar{z}, s) \\
& =s^{p} L_{j} \overline{\tilde{f}_{0 k}}(z, \bar{z}, s)+s^{2 l} h(z, \bar{z}, s),
\end{aligned}
$$

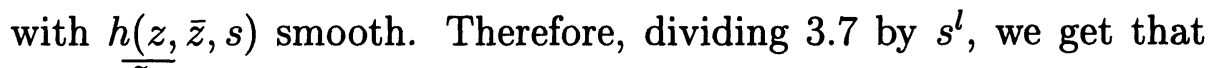
$s^{p-l} L_{j} \tilde{f}_{0 k}$ is smooth; putting $s=0$ in 3.7 , we get 3.6 . Hence the claim is proved. On the other hand, as $m \geq 1$, we have

$$
\begin{aligned}
{\left[L^{\alpha}\left(\operatorname{det} \overline{\tilde{f}_{j k}}\right)\right](0) } & =\left[L^{\alpha}\left(\operatorname{det} \overline{\tilde{f}_{j k}}(z, \bar{z}, 0)\right)\right](0) \\
& =\left[D^{\alpha}\left(\operatorname{det} \overline{\tilde{f}_{j k}}(z, \bar{z}, 0)\right)\right](0)
\end{aligned}
$$

where

$$
D^{\alpha}=\frac{\partial^{\alpha_{1}}}{\partial \bar{z}_{1}^{\alpha_{1}}} \ldots \frac{\partial^{\alpha_{n}}}{\partial \bar{z}_{n}^{\alpha_{n}}} .
$$

Using 2.18, we have, for $\bar{w}=s-i s^{m^{\prime}} \tilde{\varphi}(z, \bar{z}, s)$,

$$
\begin{aligned}
\overline{\tilde{f}_{j k}}(z, \bar{z}, 0)= & \left(\frac{L_{j} \overline{\tilde{f}_{0 k}}}{s^{l-p}}\right)(z, \bar{z}, 0) \\
\sim & \left(s^{p-l}\left[L_{j}\left(s^{-p}\left(\bar{F}_{p k}^{*} \bar{w}^{p}+\ldots+\bar{F}_{l k}^{*} \bar{w}^{l}+\ldots\right)\right)\right]\right)(z, \bar{z}, 0) \\
\equiv & \left(p s^{p-l} \bar{F}_{p k}^{*}\left(1-i s^{m-1} \tilde{\varphi}\right)^{p-1}\left(-i s^{m-1} \tilde{\varphi}_{\bar{z}_{j}}\right)\right)(z, \bar{z}, 0)+\ldots \\
& +\left(l \bar{F}_{l k}^{*}\left(1-i s^{m-1} \tilde{\varphi}\right)^{l-1}\left(-i s^{m-1} \tilde{\varphi}_{\bar{z}_{j}}\right)\right. \\
& \left.+\frac{\partial \bar{F}_{l k}^{*}}{\partial \bar{z}_{j}}(\bar{z})\left(1-i s^{m-1} \tilde{\varphi}\right)^{l}\right)(z, \bar{z}, 0) \equiv \frac{\partial \bar{F}_{l k}^{*}}{\partial \bar{z}_{j}}(\bar{z}) .
\end{aligned}
$$

Therefore,

$$
L^{\alpha}\left(\operatorname{det} \overline{\tilde{f}_{j k}}\right)(0)=\left[D^{\alpha}\left(\operatorname{det} \frac{\partial \bar{F}_{l k}^{*}}{\partial \bar{z}_{j}}\right)\right](0)
$$

By assumption, we have

$$
\operatorname{det}\left(\frac{\partial \bar{F}_{l k}^{*}}{\partial \bar{z}_{j}}(\bar{z})\right) \not \equiv 0
$$


Using 3.9 and 3.10, we get the desired conclusion 3.2. On the other hand,

$$
D(z, \bar{z}, s)=\operatorname{det} L_{j} \bar{f}_{k}(z, \bar{z}, s)=\operatorname{det} s s^{l} \tilde{\tilde{f}}_{j k}(z, \bar{z}, s)=s^{n l}\left(\operatorname{det} \overline{\tilde{f}}_{j k}\right)(z, \bar{z}, s) .
$$

Hence, we have

$$
\begin{aligned}
L^{\alpha} D(z, \bar{z}, s) & =L^{\alpha}\left(s^{n l}\left(\operatorname{det} \overline{\tilde{f}_{j k}}\right)\right)(z, \bar{z}, s) \\
& =s^{n l}\left(L^{\alpha}\left(\operatorname{det} \overline{\tilde{f}_{j k}}\right)(z, \bar{z}, s)+s k(z, \bar{z}, s)\right),
\end{aligned}
$$

with $k(z, \bar{z}, s)$ smooth. Using 3.2 , we get the desired conclusion 3.4.

Let $M$ given in normal coordinates by 1.2. Solving for $\bar{w}$ in 1.2 , it is easily shown that $M$ is also given by

$$
\bar{w}=Q(z, \bar{z}, w)=w+w^{m} S(z, \bar{z}, w)=w+\sum_{j=m}^{\infty} R_{j}(z, \bar{z}) w^{j},
$$

with $R_{j}(z, 0) \equiv R_{j}(0, \bar{z}) \equiv 0$.

Let $k_{0}$ be defined by 1.9 and $g$ be the CR function obtained from $\mathcal{G}$ by restriction to $M$. We need the following lemmas:

LEMma 3.12. Let $\left(M, M^{\prime}, \mathcal{H}\right)$ satisfy the hypothesis of the reflection principle, where $M^{\prime}$ is of infinite type at 0 and $G \not \equiv 0$. Then we have

$$
g(z, \bar{z}, s)=s^{k_{0}} g_{1}(z, \bar{z}, s),
$$

with $g_{1}(z, \bar{z}, s)$ smooth and $g_{1}(0) \neq 0$.

Proof. See Proof of Theorem 3 in [5].

LEMma 3.14. Let $\left(M, M^{\prime}, \mathcal{H}\right)$ satisfy the hypothesis of the reflection principle, where $M^{\prime}$ is of infinite type and $G \not \equiv 0$. If $h(z, \bar{z}, s+i t)$ is holomorphic in $R=\{s+i t$ such that $|s|<r, 0<$ $t<r\}, C^{\infty}$ in $R \cup(-r, r)$ for $|z|<\epsilon$, if $\frac{h}{g}(z, \bar{z}, s)$ is $C^{\infty}(-r, r)$ for $|z|<\epsilon$, then $\frac{h}{g}(z, \bar{z}, s)$ extends to $R$, for $|z|<\epsilon$.

Using Lemma 3.12, the proof of Lemma 3.14 is similar to that of Corollary 4.8 in [5]. We shall say that a function $t(z, \bar{z}, s)$ extends 
down (resp. up) if $s \rightarrow t(z, \bar{z}, s)$ extends continuously to a function $s+i t \rightarrow T(z, \bar{z}, s+i t)$, holomorphically for small $t<0$ (resp. small $t>0$ ), uniformly in $z$.

We have the following proposition:

Proposition 3.15. Let $\left(M, M^{\prime}, \mathcal{H}\right)$ satisfy the hypothesis of the reflection principle. If either

(1) $\mathcal{H}$ is not totally degenerate at 0 , or

(2) $M$ is not flat, $M^{\prime}$ is of infinite type at 0 given by (3.11), $G \not \equiv 0$ and

$$
\operatorname{det}\left(\frac{\partial F_{l j}^{*}}{\partial z_{k}}(z)\right) \not \equiv 0,
$$

then $R_{m^{\prime} \zeta^{\prime \alpha}}(f, \bar{f})$ satisfies the following equation:

$$
R_{m^{\prime} \zeta^{\prime \alpha}}(f, \bar{f})+K_{\alpha}(f, u)=0
$$

where $\alpha$ is any multi-index, with $|\alpha| \geq 1, K_{\alpha}$ is holomorphic at $(0, u(0)), u$ is a set of functions which extend down, and $K_{\alpha}(Z, u(0))$ $\equiv 0, Z \in \mathbb{C}^{n}$.

Proof. We shall prove Proposition 3.15 in the case (2). As $\mathcal{H}(M) \subset$ $M^{\prime}$, we have

$$
\bar{g}=g+g^{m^{\prime}} S(f, \bar{f}, g) .
$$

First, consider the case $|\alpha|=1$. Applying $L_{j}, j=1, \ldots, n$, to 3.17, and using the fact that $L_{j} g=L_{j} f_{k}=0, k=1, \ldots, n$, we get

$$
L_{j} \bar{g}=\sum_{k=1}^{n} g^{m^{\prime}} S_{\zeta_{k}^{\prime}}(f, \bar{f}, g) L_{j} \bar{f}_{k} .
$$

Considering the $n$ equations 3.18 with unknown $g^{m^{\prime}} S_{\zeta_{k}^{\prime}}(f, \bar{f}, g)$ and using Cramer's rule, we obtain

$$
D g^{m^{\prime}} S_{\zeta_{k}^{\prime}}(f, \bar{f}, g)=h_{k}
$$

where $h_{k}$ extends down, and $D$ given by 3.3. Choose $\beta_{0}$ of minimal length satisfying 3.4. Taking the complex conjugate of 3.17 , and raising to the $m^{\prime}$ th power, we obtain

$$
g^{m^{\prime}}=\bar{g}^{m^{\prime}}\left(1+\bar{g}^{m^{\prime}-1} \bar{S}(\bar{f}, f, \bar{g})\right)^{m^{\prime}} .
$$


Replacing 3.20 in 3.19 , and using Lemma 3.14, we obtain

$$
\begin{aligned}
& D\left(1+\bar{g}^{m^{\prime}-1} \bar{S}(\bar{f}, f, \bar{g})\right)^{m^{\prime}} S_{\zeta_{k}^{\prime}}(f, \bar{f}, g) \\
& =\frac{h_{k}}{\bar{g}^{m^{\prime}}}=\tilde{h}_{k}, \tilde{h}_{k} \text { extending down. }
\end{aligned}
$$

Applying $L^{\beta_{0}}$ to both sides of 3.21 , we get

$$
\begin{aligned}
& \left(L^{\beta_{0}} D\right)\left(1+\bar{g}^{m^{\prime}-1} \bar{S}(\bar{f}, f, \bar{g})\right)^{m^{\prime}} S_{\zeta_{k}^{\prime}}(f, \bar{f}, g) \\
& +\sum_{\gamma_{1}+\gamma_{2}=\beta_{0}, \gamma_{2} \neq 0}\left(L^{\gamma_{1}} D\right)\left(L^{\gamma_{2}}\left(\left(1+\bar{g}^{m^{\prime}-1} \bar{S}(\bar{f}, f, \bar{g})\right)^{m^{\prime}} S_{\zeta_{k}^{\prime}}(f, \bar{f}, g)\right)\right) \\
& \quad=L^{\beta_{0}} \tilde{h}_{k} .
\end{aligned}
$$

Dividing 3.22 by $L^{\beta_{0}} D=s^{n l} D_{\beta_{0}}$, we get

$$
\begin{aligned}
& \left(1+\bar{g}^{m^{\prime}-1} \bar{S}(\bar{f}, f, \bar{g})\right)^{m^{\prime}} S_{\zeta_{k}^{\prime}}(f, \bar{f}, g) \\
& +\sum_{\gamma_{1}+\gamma_{2}=\beta_{0}, \gamma_{2} \neq 0}\left(\frac{L^{\gamma_{1}} D}{s^{n l} D_{\beta_{0}}}\right)\left(L^{\gamma_{2}}\left(\left(1+\bar{g}^{m^{\prime}-1} \bar{S}(\bar{f}, f, \bar{g})\right)^{m^{\prime}} S_{\zeta_{k}^{\prime}}(f, \bar{f}, g)\right)\right) \\
& \quad=\frac{L^{\beta_{0}} \tilde{h}_{k}}{s^{n l} D_{\beta_{0}}} .
\end{aligned}
$$

Taking the complex conjugate of 3.17 and using 3.11 , we get

$$
S_{\zeta_{k}^{\prime}}(f, \bar{f}, g)=R_{m^{\prime} \zeta_{k}^{\prime}}(f, \bar{f})+\bar{g} T_{k}(f, \bar{f}, \bar{g})
$$

with $T_{k}$ holomorphic near 0 . Since we work in normal coordinates,we have

$$
b(f, \bar{f}, g)=\frac{1}{\left(1+\bar{g}^{m^{\prime}-1} \bar{S}(\bar{f}, f, \bar{g})\right)^{m^{\prime}}},
$$


with $b$ holomorphic near 0 . Multiplying 3.23 by 3.25 , and making use of 3.24 , we obtain

$$
\begin{aligned}
R_{m^{\prime} \zeta_{k}^{\prime}}(f, \bar{f}) & \\
= & -b(f, \bar{f}, \bar{g})\left[\sum_{\gamma_{1}+\gamma_{2}=\beta_{0}, \gamma_{2} \neq 0}\left(\frac{L^{\gamma_{1}} D}{s^{n l} D_{\beta_{0}}}\right)\right. \\
& \left.\cdot\left(L^{\gamma_{2}}\left(\left(1+\bar{g}^{m^{\prime}-1} \bar{S}(\bar{f}, f, \bar{g})\right)^{m^{\prime}} S_{\zeta_{k}^{\prime}}(f, \bar{f}, g)\right)\right)\right] \\
& +b(f, \bar{f}, \bar{g})\left[\frac{L^{\beta_{0}} \tilde{h}_{k}}{s^{n l} D_{\beta_{0}}}\right]-\bar{g} T_{k}(f, \bar{f}, g) .
\end{aligned}
$$

Put

$$
u=\left\{\frac{L^{\gamma_{1}} D}{s^{n l} D_{\beta_{0}}}, \bar{f}, \bar{g}, L^{\alpha} \bar{f}, \mathrm{E}^{\beta} \bar{g}, \frac{L^{\beta_{0}} \tilde{h}_{k}}{s^{n l} D_{\beta_{0}}},|\alpha|,|\beta| \leq \beta_{0}\right\} .
$$

By 3.23,

$$
\frac{L^{\beta_{0}} \tilde{h}_{k}}{s^{n l} D_{\beta_{0}}}
$$

is smooth and hence extends down by Lemma 3.14. Hence, $u$ is a set of functions which extend down. Using 3.23 , the minimality of $\beta_{0}$, we obtain that

$$
\frac{L^{\beta_{0}} \tilde{h}_{k}}{s^{n l} D_{\beta_{0}}}(0)=0
$$

Using the minimality of $\beta_{0}, 3.26$, and 3.27 , we obtain the desired conclusion 3.16 for $|\alpha|=1$. Consider the case $|\alpha|=2$. Dividing 3.19 by $D$, and applying $L_{j}, j=1, \ldots, n$, to both sides of the obtained equation, we get

$$
L_{j}\left(g^{m^{\prime}} S_{\zeta_{k}^{\prime}}(f, \bar{f}, g)\right)=L_{j} \frac{h_{k}}{D}=\frac{h_{k j}^{*}}{D^{2}}
$$

with $h_{k j}^{*}$ smooth, extending down. Therefore

$$
g^{m^{\prime}} \sum_{p=1}^{n} S_{\zeta_{k}^{\prime} \zeta_{p}^{\prime}}(f, \bar{f}, g) L_{j} \bar{f}_{p}=\frac{h_{k j}^{*}}{D^{2}} .
$$


Hence, by Cramer's rule, $D g^{m^{\prime}} S_{\zeta_{k}^{\prime} \zeta_{p}^{\prime}}(f, \bar{f}, g)=\frac{a_{k p}}{D^{2}}$, with $a_{k p}$ smooth, extending down. Using 3.20, and Lemma 3.14, we obtain

$$
D^{3}\left(1+\bar{g}^{m^{\prime}-1} \bar{S}(\bar{f}, f, \bar{g})\right)^{m^{\prime}} S_{\zeta_{k}^{\prime} \zeta_{p}^{\prime}}(f, \bar{f}, g)=\tilde{a}_{k p},
$$

with $\tilde{a}_{k p}$ extending down. Define

$$
\begin{aligned}
B^{0}=\left\{\beta \in \mathbb{Z}^{n}\right. & \text { of minimal length such that } \left.D_{\beta}(0) \neq 0\right\} . \\
& \text { with } D_{\beta} \text { defined by }(3.4)
\end{aligned}
$$

Define

$$
B^{12 \ldots j}=\left\{\beta=\left(\beta_{1}, \ldots, \beta_{n}\right) \in B^{12 \ldots j-1} \text { such that } \beta_{j} \text { is minimal }\right\} .
$$

There exists $j_{0}$ such that $\left|B^{12 \ldots j_{0}}\right|=1$. Take $\beta_{0}$ to be the unique element of $B^{12 \ldots j_{0}}$. Applying $L^{3 \beta_{0}}$ to both sides of 3.28 , we get that

$$
\begin{aligned}
& \left(L^{\beta_{0}} D\right)^{3}\left(1+\bar{g}^{m^{\prime}-1} \bar{S}(\bar{f}, f, \bar{g})\right)^{m^{\prime}} S_{\zeta_{k}^{\prime} \zeta_{p}^{\prime}}(f, \bar{f}, g) \\
& \quad+\sum_{\substack{\gamma_{1}+\gamma_{2}+\gamma_{3}+\gamma_{4}=3 \beta_{0} \\
\left(\gamma_{1}, \gamma_{2}, \gamma_{3}\right) \neq\left(\beta_{0}, \beta_{0}, \beta_{0}\right)}}\left(L^{\gamma_{1}} D\right)\left(L^{\gamma_{2}} D\right)\left(L^{\gamma_{3}} D\right) L^{\gamma_{4}} \\
& \quad \cdot\left(\left(1+\bar{g}^{m^{\prime}-1} \bar{S}(\bar{f}, f, \bar{g})\right)^{m^{\prime}} S_{\zeta_{k}^{\prime} \zeta_{p}^{\prime}}(f, \bar{f}, g)\right)=L^{3 \beta_{0}} \tilde{a}_{k p} .
\end{aligned}
$$

By the choice of $\beta_{0}$, a term of the form

$$
\left(L^{\gamma_{1}} D\right)\left(L^{\gamma_{2}} D\right)\left(L^{\gamma_{3}} D\right)\left(\left(1+\bar{g}^{m^{\prime}-1} \bar{S}(\bar{f}, f, \bar{g})\right)^{m^{\prime}} S_{\zeta_{k}^{\prime} \zeta_{p}^{\prime}}(f, \bar{f}, g)\right)
$$

with $\left|\gamma_{1}\right|=\left|\gamma_{2}\right|=\left|\gamma_{3}\right|=\left|\beta_{0}\right|$ cannot occur unless $\gamma_{1}=\gamma_{2}=\gamma_{3}=\beta_{0}$. That means that

$$
\frac{\left(L^{\gamma_{1}} D\right)\left(L^{\gamma_{2}} D\right)\left(L^{\gamma_{3}} D\right)}{\left(L^{\beta_{0}} D\right)^{3}}(0)=0, \gamma_{1}+\gamma_{2}+\gamma_{3}+\gamma_{4}=3 \beta_{0},
$$

by 3.4 and by minimality of $\beta_{0}$. We divide both sides of 3.29 by $\left(L^{\beta_{0}} D\right)^{3}$ and we put

$$
\begin{aligned}
u=\left\{\frac{\left(L^{\gamma_{1}} D\right)\left(L^{\gamma_{2}} D\right)\left(L^{\gamma_{3}} D\right)}{\left(L^{\beta_{0}} D\right)^{3}}, \bar{f}, \bar{g}, L^{\alpha} \bar{f}, L^{\beta} \bar{g}\right. & \\
& \left.\frac{L^{3 \beta_{0}} \tilde{a}_{k p}}{\left(L^{\beta_{0}} D\right)^{3}},|\alpha|,|\beta| \leq\left|3 \beta_{0}\right|\right\} .
\end{aligned}
$$


We can apply the same process as for $|\alpha|=1$, in order to get the desired conclusion for the case $|\alpha|=2$. For the general case, i.e. for a multi-index $\alpha$ of any length, we observe that

$$
g^{m^{\prime}} S_{\zeta^{\prime \alpha}}(f, \bar{f}, g)=\frac{d_{\alpha}}{D^{2|\alpha|-1}}
$$

with $d_{\alpha}$ extending down. We apply the same proof as in the case of $|\alpha|=1,2$ to get the desired conclusion 3.16. This completes the proof of Proposition 3.15.

We need the following lemma:

LemMA 3.30. Let $M$ be given by (3.11). Suppose that $M$ is $m$ essential. Write

$$
R_{m}(z, \bar{z})=\sum_{\alpha} b_{\alpha}(z) \bar{z}^{\alpha}
$$

Then for every $z_{0}$ sufficiently small, there exists a multi-index $\alpha_{0}$ such that $b_{\alpha_{0}}\left(z_{0}\right) \neq 0$.

The proof is left to the reader.

Proposition 3.31. Let $\left(M, M^{\prime}, \mathcal{H}\right)$ satisfy the hypothesis of the reflection principle, with $M$ non flat, $M^{\prime} m^{\prime}$-essential at 0 , of infinite type, $\operatorname{det}\left(\frac{\partial F_{l j}^{*}}{\partial z_{k}}(z)\right) \not \equiv 0$ and $G \not \equiv 0$. Then each $f_{j}, j=1, \ldots, n$, satisfies a polinomial equation with analytic coefficients depending on functions which extend down.

Proof. Let $M^{\prime}$ given in normal coordinates by 3.11. Using Lemma 3.30 and the Nullstellensatz, we can find $N$ and $r$ such that

$$
z_{j}^{\prime N}=\sum_{\alpha=1}^{r} c_{j \alpha}\left(z^{\prime}\right) b_{\alpha}\left(z^{\prime}\right), j=1, \ldots, n
$$

with $c_{j \alpha}\left(z^{\prime}\right)$ convergent power series. We also have

$$
R_{m^{\prime} \zeta^{\prime \alpha}}\left(z^{\prime}, \bar{z}^{\prime}\right)=\alpha ! b_{\alpha}\left(z^{\prime}\right)+\sum_{|s| \geq 1} d_{\alpha s}\left(z^{\prime}\right){\overline{z^{\prime}}}^{s}
$$

with $d_{\alpha s}\left(z^{\prime}\right)$ convergent power series. As $f_{j}(0)=0$, we can substitute $f_{j}$ in 3.32 and we get

$$
f_{j}^{N}=\sum_{\alpha=1}^{r} c_{j \alpha}(f) b_{\alpha}(f)
$$


Using Proposition 3.15, 3.33 and 3.34, we obtain

$$
f_{j}^{N}+H_{j}(f, u)=0,
$$

with $H_{j}$ holomorphic at $(0, u(0)), H_{j}(Z, u(0)) \equiv 0, Z \in \mathbb{C}^{n}$, and $u$ a set of functions which extend down. Using 3.35, the Weierstrass Preparation Theorem and the classical Newton's Theorem for symmetric functions, we claim that $f_{j}, 1 \leq j \leq n$, satisfies a polynomial equation with holomorphic coefficients depending on the set of functions $u$ which extend down. The proof of the claim follows by inspecting the very end of Lemma 6.1 in [3]. Hence, we get the desired conclusion.

We have the following proposition:

Proposition 3.36. Let $\left(M, M^{\prime}, \mathcal{H}\right)$ satisfy the hypothesis of the reflection principle. If either

(1) $\mathcal{H}$ is not totally degenerate at 0 , or

(2) $M$ is not flat, $M^{\prime}$ is of infinite type at 0 given by (3.11), $G \not \equiv 0$ and $\operatorname{det}\left(\frac{\partial F_{l j}^{*}}{\partial z_{k}}(z)\right) \not \equiv 0$,

then there exists $r>0$ such that for every $z_{0} \in \mathbb{C}^{n}$ fixed, $\left|z_{0}\right|<r$ and every multi-index $\alpha$, there exist functions $a(s+i t)$ and $b(s+i t)$ holomorphic in the domain

$$
R=\{s+i t \text { such that }|s|<r,-r<t<0\},
$$

smooth in $R \bigcup(-r,+r)$, such that

$$
Q_{\zeta^{\alpha}}(f, \bar{f}, g)\left(z_{0}, \bar{z}_{0}, s\right)=\frac{a(s)}{b(s)},|s|<r .
$$

The proof is similar to that of Lemma 5.3 in [3] and is left to the reader.

Proof of Theorem 1 and Theorem 3. By Proposition 2.11, (1) implies (2) in Theorem 1. Therefore we only have to prove Theorem 1 for condition (2) or (3). As 1.7 implies 1.6, we only have to prove Theorem 1 for condition (3) and Theorem 3 for condition (2). Using Proposition 3.31 and Lemma 7.1 in [3], we conclude that for each 
$\alpha, Q_{\zeta^{\alpha}}(f, \bar{f}, g)$ satisfies a polynomial relation with coefficients which are analytic functions depending on functions which extend down. Using Proposition 3.36, Lemma 7.1 in [3] and Lemma 8.15 in [1], we conclude that $Q_{\zeta^{\alpha}}(f, \bar{f}, g)$ extends down for every $\alpha$, and that

$$
\left|Q_{\zeta^{\alpha}}(f, \bar{f}, g)(z, \bar{z}, s+i t)\right| \leq C^{\alpha} \alpha !
$$

Therefore, following the proof of Theorem 1 in [3], we can conclude that

$$
Q(f, \lambda, g)(z, \bar{z}, s)=\sum_{\alpha=0}^{\infty} \frac{(\lambda-\bar{f})^{\alpha}}{\alpha !} Q_{\zeta^{\alpha}}(f, \bar{f}, g)(z, \bar{z}, s)
$$

extends up and down, uniformly in $\lambda$. Taking $\lambda=0$, we get that $g$ extends down, as we work in normal coordinates. Consider

$$
\frac{Q(f, \lambda, g)-g}{g^{m^{\prime}}}=S(f, \lambda, g)
$$

By Lemma 3.14, $S(f, \lambda, g)$ extends up and down. Again, inspecting the proof of Theorem 1 in [3], using the Weierstrass Preparation Theorem, we obtain that $f$ extends down. We are able to complete the proof of (3) of Theorem 1 and the proof of (2) of Theorem 3 by using the following Criterion proved in [2]: $\mathcal{H}$ extends holomorphically through 0 in $\mathbb{C}^{n+1}$ if and only if the function $s \rightarrow H(z, \bar{z}, s)$ extends holomorphically through 0 in $\mathbb{C}$, uniformly in $z$.

\section{Proof of Theorem 2 and Theorem 4.}

Proof of Theorem 2. The case $m=0$ has been considered in [3] and [4]. Assume $m>0$. Using Propositions 2.2 and 2.11, Theorem 2 is proved for condition (1). We have to prove Theorem 2 under condition (2). Inspecting the proof of Proposition 2.11, and using Proposition 2.2, we conclude that

$$
h(z, \bar{z}) \varphi(z, \bar{z}, 0) \equiv \psi(F(z, 0), \bar{F}(\bar{z}, 0), 0),
$$

with $h(z, \bar{z})$ a formal power series such that $h(0) \neq 0$. Inspecting the proof of Theorem 2 in [3], which uses tools of commutative algebra, we conclude that $M$ is $m$-essential. Using condition (1), we get the desired conclusion. 
Proof of Theorem 4. By 3.11, $M^{\prime}$ can be parametrized by

$$
w^{\prime}-\bar{w}^{\prime}=\sum_{j=1}^{\infty} P_{j}\left(z^{\prime}, \bar{z}^{\prime}\right) \bar{w}^{\prime}
$$

with $P_{j}\left(z^{\prime}, 0\right) \equiv P_{j}\left(0, \bar{z}^{\prime}\right) \equiv 0 . M$ can be parametrized by

$$
w=\bar{w}\left(1+\bar{w}^{m-1} R(z, \bar{z}, \bar{w})\right)=s \lambda,
$$

where $s=\bar{w}$ and $\lambda=1+\bar{w}^{m-1} R(z, \bar{z}, \bar{w})$, with

$$
R(0, \bar{z}, \bar{w}) \equiv R(z, 0, \bar{w}) \equiv 0 .
$$

As $\mathcal{H}(M) \subset M^{\prime}$, we have

(4.2) $G(z, s \lambda)-\bar{G}(\bar{z}, s) \equiv \sum_{j=1}^{\infty} P_{j}(F(z, s \lambda), \bar{F}(\bar{z}, s))(\bar{G}(\bar{z}, s))^{j}$.

Putting $\bar{z}=0$ in 4.2 , we get

$$
G(z, s) \equiv \bar{G}(0, s)+\sum_{j=1}^{\infty} P_{j}(F(z, s), \bar{F}(0, s)) \bar{G}(0, s)^{j}
$$

Taking the complex conjugate of 4.3 , we get ( $s$ taken to be real)

$$
\bar{G}(z, s) \equiv G(0, s)+\sum_{j=1}^{\infty} \bar{P}_{j}(\bar{F}(\bar{z}, s), F(0, s)) G(0, s)^{j} .
$$

Substituting for $\bar{G}(\bar{z}, s)$ in the right hand side of 4.2 , we get

$$
\begin{aligned}
G(z, s \lambda)-\bar{G}(\bar{z}, s) & \\
\equiv & \sum_{j=1}^{\infty} P_{j}(F(z, s \lambda), \bar{F}(\bar{z}, s))(G(0, s) \\
& \left.+\sum_{q=1}^{\infty} \bar{P}_{q}(\bar{F}(\bar{z}, s), F(0, s)) G(0, s)^{q}\right)^{j} .
\end{aligned}
$$

From 4.4, we get

$$
\begin{aligned}
(s \lambda)^{k_{0}} G^{k_{0}}(z, s \lambda)-s^{k_{0}} \bar{G}^{k_{0}}(\bar{z}, s) & \\
\equiv & \sum_{j=1}^{\infty} P_{j}(F(z, s \lambda), \bar{F}(\bar{z}, s))\left(s^{k_{0}} G^{k_{0}}(0, s)\right. \\
& \left.+\sum_{q=1}^{\infty} \bar{P}_{q}(\bar{F}(\bar{z}, s), F(0, s)) s^{k_{0} q} G^{k_{0}}(0, s)^{q}\right)^{j} .
\end{aligned}
$$


By the binomial formula,

(4.6) $\left(s+s^{m} R\right)^{k_{0}} G^{k_{0}}(z, s \lambda)$

$$
\equiv s^{k_{0}} G^{k_{0}}(z, s \lambda)+C s^{m+k_{0}-1} R\left(G^{k_{0}}(z, s \lambda)+\alpha(z, \bar{z}, s)\right) \text {, }
$$

with $C$ a constant different from 0 , and $\alpha(z, \bar{z}, s)$ another formal power series such that $\alpha(0, \bar{z}, s) \equiv \alpha(z, 0, s) \equiv 0$. Hence, we get from 4.5

$$
\begin{aligned}
& s^{k_{0}} G^{k_{0}}(z, s \lambda)-s^{k_{0}} \bar{G}^{k_{0}}(\bar{z}, s) \\
& +C s^{m+k_{0}-1} R\left(G^{k_{0}}(z, s \lambda)+\alpha(z, \bar{z}, s)\right) \\
& \equiv \sum_{j=1}^{\infty} P_{j}(F(z, s \lambda), \bar{F}(\bar{z}, s))\left(s^{k_{0}} G^{k_{0}}(0, s)\right. \\
& \left.\quad+\sum_{q=1}^{\infty} \bar{P}_{q}(\bar{F}(\bar{z}, s), F(0, s)) s^{k_{0} q} G^{k_{0}}(0, s)^{q}\right)^{j}
\end{aligned}
$$

On the other hand,by Taylor's expansion, we have

$$
F(z, s \lambda) \equiv F(z, s)+s^{m} R(z, \bar{z}, s) T(z, \bar{z}, s),
$$

with $T$ another formal power series. Therefore, 4.7 becomes

$$
\begin{aligned}
& s^{k_{0}} G^{k_{0}}(z, s \lambda)-s^{k_{0}} \bar{G}^{k_{0}}(\bar{z}, s) \\
& +C s^{m+k_{0}-1} R\left(G^{k_{0}}(z, s \lambda)+\alpha(z, \bar{z}, s)\right) \\
& \equiv \sum_{j=1}^{\infty} P_{j}(F(z, s), \bar{F}(\bar{z}, s))\left(s^{k_{0}} G^{k_{0}}(0, s)\right. \\
& \left.\quad+\sum_{q=1}^{\infty} \bar{P}_{q}(\bar{F}(\bar{z}, s), F(0, s)) s^{k_{0} q} G^{k_{0}}(0, s)^{q}\right)^{j} \\
& +\sum_{j=1}^{\infty} s^{m} \tilde{T}_{j}(z, \bar{z}, s)\left(s^{k_{0}} G^{k_{0}}(0, s)\right. \\
& \left.\quad+\sum_{q=1}^{\infty} \bar{P}_{q}(\bar{F}(\bar{z}, s), F(0, s)) s^{k_{0} q} G^{k_{0}}(0, s)^{q}\right)^{j}
\end{aligned}
$$


where $\tilde{T}_{j}$ is another formal power series. We can rewrite 4.9 as

$$
\begin{aligned}
& \text { (4.10) } s^{k_{0}} G^{k_{0}}\left(z, s+s^{m} R\right)-s^{k_{0}} \bar{G}^{k_{0}}(\bar{z}, s) \\
& +C s^{m+k_{0}-1} R\left(G^{k_{0}}(z, s \lambda)\right. \\
& +\alpha(z, \bar{z}, s))+s^{m+k_{0}} U(z, \bar{z}, s) \\
& \equiv \sum_{j=1}^{\infty} P_{j}(F(z, s), \bar{F}(\bar{z}, s))\left(s^{k_{0}} G^{k_{0}}(0, s)\right. \\
& \left.\quad+\sum_{q=1}^{\infty} \bar{P}_{q}(\bar{F}(\bar{z}, s), F(0, s)) s^{k_{0} q} G^{k_{0}}(0, s)^{q}\right)^{j}
\end{aligned}
$$

with $U(z, \bar{z}, s)$ another formal power series.

Write $F(z, s)=a_{p} s^{p}+\ldots+F_{l}^{*}(0) s^{l}+s^{l} \mathcal{F}^{*}$, with $\mathcal{F}^{*}(z, s)=$ $F_{l}^{*}(z)-F_{l}^{*}(0)+s \gamma$, where $\gamma$ is another formal power series. Therefore the right hand side of 4.10 can be written as

$$
s^{n_{1}} h_{1}(s)+s^{n_{2}} h_{2}\left(\mathcal{F}^{*}, \overline{\mathcal{F}^{*}}, s\right)+s^{n_{3}} h_{3}\left(\mathcal{F}^{*}, \overline{\mathcal{F}^{*}}, s\right)
$$

where $h_{1}, h_{2}, h_{3}$ are formal power series and $h_{1}(0) \neq 0, h_{2}(x, y, 0) \not \equiv$ $0, h_{2}$ contains only pure power of $\mathcal{F}^{*}$ and $\overline{\mathcal{F}^{*}}, h_{3}(x, y, 0) \not \equiv 0$ and $h_{3}$ contains no pure power of $\mathcal{F}^{*}$ and $\overline{\mathcal{F}^{*}}$.

We claim that $m+k_{0}-1=n_{3}$. The proof of the claim is similar to that of (2) of Proposition 2.2 and is left to the reader. Differentiating 4.10 with respect to $s m+k_{0}-1=n_{3}$ times and putting $s=0$, we get

$$
h(z, \bar{z}, 0) R(z, \bar{z}, 0)=h_{3}\left(F_{l}^{*}(z)-F_{l}^{*}(0), \bar{F}_{l}^{*}(\bar{z})-\bar{F}_{l}^{*}(0), 0\right),
$$

with $h$ a formal power series such that $h(0) \neq 0$, by (1) of Proposition 2.2. The rest of the proof is similar to that of Proposition 2.11 and is left to the reader.

\section{REFERENCES}

[1] M. S. Baouendi, S. Bell and L. P. Rothshild, Mappings of three-dimensional CR manifolds and their holomorphic extension, Duke Math. J., 56(1988), 503-530.

[2] M. S. Baouendi, H. Jacobowitz and F. Treves, On the analyticity of CR mappings, Ann. of Math., 122 (1985), 365-400.

[3] M. S. Baouendi and L. P. Rothschild, Germs of CR maps between real analytic hypersurfaces, Invent. Math., 93 (1988), 481-500. 
[4] - Geometric properties of smooth and holomorphic mappings between hypersurfaces in complex space, J. Diff. Geom., 31 (1990), 473-499.

[5] , A General Reflection Principle in $\mathbb{C}^{2}$, J. Funct. Anal., 99 (1991), 409-442.

[6] S. Bell, Analytic hypoellipticity of the $\bar{\partial}-$ Neumann problem and extendability of holomorphic mappings, Acta Math., 147 (1981), 109-116.

[7] J. D'Angelo, The notion of formal essential finiteness for smooth real hypersurfaces, Indiana J. Math., 36 (1987), 897-903.

[8] T. Bloom and I. Graham, On type conditions for generic submanifolds of $\mathbb{C}^{n}$, Invent. Math., 40 (1977), 217-243.

[9] S. S. Chern and J. Moser, Real hypersurfaces in complex manifolds, Acta Math., 133 (1974), 219-271.

[10] K. Diederich and J. E. Fornaess, Proper holomorphic mappings between real analytic pseudoconvex domains in $\mathbb{C}^{n}$, Math. Ann., 282 (1988), 681-700.

[11] K. Diederich and S. M. Webster, A reflection principle for degenerate real hypersurfaces, Duke Math. J., 47 (1980), 835-843.

[12] D. Eisenbud and H. Levine, An algebraic formula for the degree of a $C^{\infty}$ map germ, Annals of Math., 106 (1977), 19-44.

[13] P. Griffiths and J. Harris, Principles of Algebraic Geometry, WileyInterscience, New York (1978).

[14] L. Hörmander, Hypoelliptic second order differential equations, Acta. Math., 119 (1967), 147-171.

[15] J. J. Kohn, Boundary behavior of $\bar{\partial}$ on weakly pseudoconvex manifolds of dimension two, J. Differential Geom., 6 (1972), 523-542.

[16] H. Lewy, On the boundary behavior of holomorphic mappings, Acad. Naz. Lincei, 35 (1977), 1-8.

[17] M. Nagata, Local Rings, Wiley-Interscience, New York (1962).

[18] S. I. Pincuk, On proper holomorphic mappings of strictly pseudoconvex domains, Siberian Math. J., 15 (1974), 909-917.

[19] O. Zariski and P. Samuel, Commutative Algebra, Van Nostrand, New York 1-2 (1958-1960).

Received July 29, 1992 and in revised form February 3, 1993.

\section{UNIVERSITE DE GENEVE}

2-4 RUE DE LIEVRE CP 240

1211 Geneve, Switzerland

E-mail address: Francine.Meylan@ima.unil.ch 


\title{
PACIFIC JOURNAL OF MATHEMATICS
}

Founded by E. F. Beckenbach (1906-1982) and F. Wolf (1904-1989)

\section{EDITORS}

Sun-Yung Alice Chang (Managing Editor)

University of California

Los Angeles, CA 90095-1555

pacific@math.ucla.edu

F. Michael Christ

University of California

Los Angeles, CA 90095-1555

christ@math.ucla.edu

Thomas Enright

University of California

San Diego, La Jolla, CA 92093

tenright@ucsd.edu

Nicholas Ercolani

University of Arizona

Tucson, AZ 85721

ercolani@math.arizona.edu
Robert Finn

Stanford University

Stanford, CA 94305

finn@gauss.stanford.edu

Vaughan F. R. Jones

University of California

Berkeley, CA 94720

vfr@math.berkeley.edu

Steven Kerckhoff

Stanford University

Stanford, CA 94305

spk@gauss.stanford.edu
Martin Scharlemann

University of California

Santa Barbara, CA 93106

mgscharl@math.ucsb.edu

\section{Gang Tian}

Courant Institute

New York University

New York, NY 10012-1100

tiang@taotao.cims.nyu.edu

V. S. Varadarajan

University of California

Los Angeles, CA 90095-1555

vsv@math.ucla.edu

\section{SUPPORTING INSTITUTIONS}

\section{CALIFORNIA INSTITUTE OF TECHNOLOGY \\ NEW MEXICO STATE UNIVERSITY \\ OREGON STATE UNIVERSITY \\ STANFORD UNIVERSITY \\ UNIVERSITY OF ARIZONA \\ UNIVERSITY OF BRITISH COLUMBIA \\ UNIVERSITY OF CALIFORNIA UNIVERSITY OF HAWAII}

\author{
UNIVERSITY OF MONTANA \\ UNIVERSITY OF NEVADA, RENO \\ UNIVERSITY OF OREGON \\ UNIVERSITY OF SOUTHERN CALIFORNIA \\ UNIVERSITY OF UTAH \\ UNIVERSITY OF WASHINGTON \\ WASHINGTON STATE UNIVERSITY
}

The supporting Institutions listed above contribute to the cost of publication of this Journal, but they are not owners or publishers and have no responsibility for its contents or policies.

Manuscripts must be prepared in accordance with the instructions provided on the inside back cover.

The Pacific Journal of Mathematics (ISSN 0030-8730) is published monthly except for July and August. Regular subscription rate: $\$ 215.00$ a year (10 issues). Special rate: $\$ 108.00$ a year to individual members of supporting institutions.

Subscriptions, orders for back issues published within the last three years, and changes of subscribers address should be sent to Pacific Journal of Mathematics, P.O. Box 4163, Berkeley, CA 94704-0163, U.S.A. Prior back issues are obtainable from Kraus Periodicals Co., Route 100, Millwood, NY 10546.

The Pacific Journal of Mathematics at the University of California, c/o Department of Mathematics, 981 Evans Hall, Berkeley, CA 94720 (ISSN 0030-8730) is published monthly except for July and August. Second-class postage paid at Berkeley, CA 94704, and additional mailing offices. POSTMASTER: send address changes to Pacific Journal of Mathematics, P.O. Box 6143, Berkeley, CA 94704-0163.

\author{
PUBLISHED BY PACIFIC JOURNAL OF MATHEMATICS at University of California, \\ Berkeley, CA 94720, A NON-PROFIT CORPORATION \\ This publication was typeset using AMS-LATEX, \\ the American Mathematical Society's TEX macro system. \\ Copyright (C) 1995 by Pacific Journal of Mathematics
}




\section{PACIFIC JOURNAL OF MATHEMATICS}

Volume 169 No. $1 \quad$ May 1995

Minimal sets of periods for torus maps via Nielsen numbers

Lluís AlsedÀ, StewART BALdWin, JAUME Llibre,

RICHARD SWANSON and WIESLAW SZLENK

Diagonalizing Hilbert cusp forms

Timothy ATWILL

A splitting criterion for rank 2 vector bundles on $\mathbf{P}^{n}$

EDOARDO BALLICO

Controlling Tietze-Urysohn extensions

MARC FRANTZ

Length of Julia curves

DAVID H. HAMILTON

On the uniqueness of capillary surfaces over an infinite strip

JENN-FANG HWANG

Volume estimates for log-concave densities with application to iterated 107 convolutions

MARIUS JUNGE

A reflection principle in complex space for a class of hypersurfaces and mappings

FRANCINE ANTOINETTE MEYLAN

Jean Bourgain's analytic partition of unity via holomorphic martingales 161

PAUL F.X. MÜLLER

Characters of Brauer's centralizer algebras 